\title{
Atuação fonoaudiológica na Unidade De Terapia Intensiva em bebê com síndrome de pterígeo poplíteo
}

\author{
Speech-language therapy at the Neonatal Intensive Care Unit with a baby \\ with popliteal pterygium syndrome
}

\author{
Susana Elena Delgado ${ }^{1}$
}

\begin{abstract}
RESUMO
O objetivo deste estudo foi descrever a intervenção fonoaudiológica para a adequação da função alimentar por meio da avaliação e tratamento do sistema estomatognático e suas funções. O estudo foi feito a partir do relato de caso de um recém-nascido portador da Síndrome de Pterígio Poplíteo, que foi alimentado por sonda, atendido na Unidade de Tratamento Intensivo Neonatal. A avaliação fonoaudiológica apresentou os seguintes resultados: mandíbula retraída com pouca abertura, gengivas superiores e inferiores hipertróficas, palato com fissura pós-forame incisivo incompleta, reflexos orais de alimentação (busca, sucção, deglutição) presentes e adequados; reflexos de defesa (mordida, gag) anteriorizados e exacerbados. Apresentou pouca tolerância ao toque intra-oral. A sucção não nutritiva teve como resultado um número de sucções/pausas de 3:1, com ritmo presente, com força débil. Na sucção nutritiva, por meio da mamadeira, apresentou sucções/pausa de 5:1, com pausas longas. Foram observados sinais de estresse de alteração respiratória, estridor inspiratório leve, um episódio de engasgo e reflexo de gag anteriorizado e exacerbado, demonstrando hipersensibilidade oral em resposta ao bico convencional da mamadeira. Foram realizadas cinco sessões de intervenção fonoaudiológica, com orientações para a mãe e a equipe de enfermagem sobre postura, estimulação oral e uso de bico ortodôntico. O recém-nascido teve alta com via oral plena e com bom ganho de peso.
\end{abstract}

Descritores: Recém-nascido; Comportamento alimentar; Anormalidades múltiplas; Fissura palatina; Terapia intensiva neonatal

\section{INTRODUÇÃO}

A assistência ao recém-nascido (RN) em fase hospitalar, quanto ao seu desenvolvimento global e alimentar, tem sido cada vez mais, campo de atuação fonoaudiológica na avaliação e tratamento dos distúrbios iniciais. Hoje em dia acompanhamos a evolução de neonatos de risco, que nascem em condições clínicas instáveis, com grau variado de dificuldades anatômicas e funcionais. Eles completam seu desenvolvimento sob condições não fisiológicas, hospitalizados, muitas vezes, por longos períodos, com grandes riscos de alterações temporárias e/ou permanentes ${ }^{(1)}$.

A alimentação por via oral é a mais adequada para a estimulação do desenvolvimento das estruturas do sistema estomatognático; porém, existem situações em que, para garantir a sobrevivência de recém-nascidos patológicos, se faz necessária a utilização de vias alternativas de alimentação: via parenteral, enteral e/ou gavagem.

(1) Mestre; Professora Adjunto do Curso de Fonoaudiologia da Universidade Luterana do Brasil - ULBRA - Canoas (RS), Brasil.

Endereço para correspondência: Susana Elena Delgado. R. Pedro Chaves Barcelos, 892/201, Bairro Bela Vista, Porto Alegre - RS, CEP: 90450-010. E-mail: sudel.ez@terra.com.br

Recebido em: 22/2/2008; Aceito em: 26/7/2008
A nutrição enteral é indicada para RN que são impedidos de ser alimentados por via oral (VO) em consequência dos déficits maturacionais fisiológicos ou alterações anatômicas, sendo necessária a utilização de sondas nasogástricas (SNG) ou orogástricas (SOG), pelas quais o alimento é depositado diretamente no tubo digestivo do $\mathrm{RN}^{(1)}$. $\mathrm{O}$ uso prolongado dessas sondas pode contribuir para modificar tanto as estruturas orais no que se refere à mobilidade, à tonicidade e, principalmente, à sensibilidade oral, quanto para alterar as funções estomatognáticas $^{(2)}$.

O fonoaudiólogo é um dos profissionais capacitados para atuar em berçário neonatal com RN pré-termo e a termo que apresentam dificuldades na alimentação e que necessitam desses procedimentos. O objetivo dessa atuação visa à avaliação e ao tratamento das alterações, com ênfase na adequação do sistema estomatognático (SE), na estimulação da alimentação oral de forma segura e eficaz e na promoção do aleitamento materno $^{(1-2)}$.

Dentre os bebês de risco que são passíveis de intervenção fonoaudiológica, existe um grupo acometido por malformações craniofaciais congênitas associadas ou não a síndromes. Estas anomalias são muito diversas, têm etiologia multifatorial e podem repercutir negativamente na função alimentar.

A complexidade do contexto em que se insere o recém- 
nascido portador de anomalias craniofaciais exige de todos, familiares e profissionais da saúde envolvidos na sua assistência, conhecimento, determinação, entendimento e paciência para que se possa alcançar o fim comum, em longo prazo, que é obter um indivíduo saudável e integrado à sociedade ${ }^{(3-4)}$.

Uma síndrome rara que traz repercussões na alimentação do bebê afetado é a do Pterígio Poplíteo. Pode ser de caráter autossômico dominante, recessivo ou multifatorial e a sua principal característica é a presença de pterígio que é uma membrana de pele que liga articulações, quase sempre bilateral, ao nível de membros inferiores, estendendo-se do calcanhar à tuberosidade do ísquio e englobando o nervo ciático, com isso limitando de maneira importante os movimentos de abdução, rotação e extensão de membros inferiores. Porém, ressaltam os autores, são extremamente variáveis as diversas manifestações que compõem a síndrome ${ }^{(4-7)}$. Entre estas encontramos os critérios mínimos de diagnóstico que incluem: pterígio poplíteo, fenda labial e/ou palatal, anomalias de dedos e anomalias genitais. Ainda, pode-se ter achados de fosseta labial inferior e pterígio de membrana palpebral, anomalias cardiovasculares, respiratórias e gastrintestinais, entre ou$\operatorname{tros}^{(6-7)}$. A etiopatogênese é desconhecida. A hipótese principal é que ocorra uma alteração do desenvolvimento baseada em um desequilíbrio entre a migração do mesoderma e a proliferação do ectoderma. Não foi comprovada a prevalência ligada à raça ou ao $\operatorname{sexo}^{(6)}$.

O diagnóstico diferencial deve ser realizado e a investigação genética é indispensável, uma vez que, na transmissão autossômica recessiva, os genitores dos afetados são normais e são frequentes os casos isolados ${ }^{(4)}$.

Uma vez estabelecido o diagnóstico, a intervenção de equipe multidisciplinar se faz necessária devido à complexidade dos aspectos alterados nesta patologia ${ }^{(6-7)}$. Serão necessários tratamentos cirúrgicos, ortopédicos e de reabilitação precoces para evitar alterações do desenvolvimento que levem a limitações permanentes.

No caso de crianças que apresentam a fissura palatina associada à síndrome, a presença do fonoaudiólogo na equipe é indispensável. Os objetivos imediatos de se alimentar uma criança fissurada são os mesmos que para uma criança saudável, porém, a assistência e suporte profissional, nestes casos, são importantes para atingir uma alimentação efetiva e bem sucedida ${ }^{(8)}$.

Vários estudos constatam que crianças nascidas com fissuras podem apresentar uma grande variedade de problemas de alimentação. Inicialmente as fissuras de lábio e as lábiopalatinas podem ter as dificuldades neonatais resolvidas com intervenção precoce de equipe multidisciplinar. Já as fissuras isoladas de palato, associadas ou não a micrognatia podem apresentar maiores dificuldades e por maior tempo, requerendo cuidados especiais $^{(8-10)}$.

A dificuldade de alimentar a criança portadora de fissura de lábio e/ou palato interfere fundamentalmente nas condições clínicas da criança, uma vez que a correção cirúrgica da anomalia, geralmente, depende do estado nutricional e do seu desenvolvimento físico.

Estudos afirmam que as dificuldades de sucção e deglutição, muitas vezes, podem levar ao agravamento do estado nutricional da criança fissurada, devido à incapacidade ou insuficiência da sucção, fadiga, otites de repetição, engasgos e tosse, regurgitação nasal e problemas pulmonares, decorrentes da aspiração de alimentos ${ }^{(8-11)}$.

Na maioria dos casos, deve ser evitada a alimentação por sonda nestes bebês. Alimentar o recém-nascido precocemente por sonda nasogástrica (SNG) interfere tanto na digestão como no próprio processo de alimentação, pois a interposição da sonda na narina atua como um fator desconfortável provocando obstrução nasal, dificuldade para fechamento adequado da boca, comprometimento da sensibilidade da cavidade oral; a alimentação oferecida em intervalos regulares pela SNG não prioriza o controle da fome, da saciedade e do volume alimentar ${ }^{(1,3)}$. Não obstante, em alguns casos, poderá haver indicação da via alternativa, ficando a cargo do fonoaudiólogo realizar a passagem para a via oral o mais rapidamente e da forma mais segura possível.

A amamentação é possível em crianças com fissura de lábio e/ou palato, sendo que a técnica da alimentação vai depender da complexidade da fissura e condições da criança. $\mathrm{O}$ aleitamento materno, embora mais trabalhoso que a mamadeira, é recomendado pelo seu valor nutritivo e qualidade antibacteriana, auxiliando na prevenção de infecções, sobretudo a do ouvido médio, comuns nos portadores dessa malformação ${ }^{(1,3)}$.

Porém, os autores são unânimes em dizer que, a maioria dos portadores de fissura poderá ter que recorrer a outros métodos, como a mamadeira, para garantir o ganho de peso suficiente, na impossibilidade de um aleitamento materno adequado ${ }^{(3,8,12)}$.

O objetivo deste estudo foi descrever a intervenção fonoaudiológica para adequação da função alimentar, por meio da avaliação e tratamento das estruturas do sistema estomatognático e suas funções, a partir do relato de caso de um bebê portador da Síndrome de Pterígio Poplíteo, alimentado por sonda, internado em Unidade de Terapia Intensiva (UTI) Neonatal.

\section{APRESENTAÇÃO DO CASO CLÍNICO}

Este estudo é do tipo observacional, de caso, contemporâneo, prospectivo e está vinculado ao projeto Caracterização do desenvolvimento da alimentação de bebês de risco internados na UTI Neonatal, aprovado pelo Comitê de Ética do Grupo de Pesquisa da ULBRA, sob n ${ }^{\circ}$ 2004-303H.

As informações do paciente foram obtidas por meio de consulta no prontuário padronizado do serviço de Neonatologia, de onde foram coletados dados da história gestacional e do parto, condições do bebê ao nascer, peso, data da internação, intercorrências clínicas durante a internação, diagnósticos, uso de sondas para alimentação, condições clínicas gerais atuais (Anexo 1). A avaliação fonoaudiológica foi realizada seguindo o protocolo padronizado do serviço de Neonatologia onde foram realizadas observações pré-alimentacão: da postura; do estado de consciência; do tônus da musculatura global; da estabilidade respiratória; das estruturas do SE (lábios, língua no repouso, língua na sucção e deglutição, bochechas, mandíbula, palato duro) e dos reflexos orais (busca, sucção, deglutição, mordida, gag); da SNN antes da alimentação e durante a alimentação com sonda (sucções/pausas, ritmo, forca, pausas) 
com introdução do dedo mínimo enluvado dentro da cavidade oral do RN e por meio da chupeta ortodôntica; e da SN na mamadeira (sucções/pausas, tempo de alimentação, sinais de estresse) (Anexo 2).

A observação de assistência à alimentação foi registrada no protocolo da UTI Neonatal (Anexo 3).

O compromisso de sigilo para a utilização dos dados do prontuário usado no estudo de caso, obrigatório para pesquisa com seres humanos (Resolução $N^{\circ}$ 196/96), foi assinado pelo pesquisador responsável.

Foram realizadas cinco sessões de avaliação e intervenção fonoaudiológica. A equipe de enfermagem e a mãe foram orientadas a realizar a estimulação oral nos demais dias da semana, pelo menos em três alimentações ao longo do dia.

O recém-nascido do estudo era do sexo feminino, nascido em 25/11/2007 no hospital do município de Cachoeirinha (RS). Teve parto cesáreo de mãe com 43 anos, fumante, na quarta gestação com três partos anteriores normais. Nasceu com idade gestacional de 38 semanas, com peso de 2150 gramas, considerado pequeno para idade gestacional, e 48 $\mathrm{cm}$ de comprimento. Apresentou Apgar 10 no primeiro e no quinto minuto, sendo transferido de hospital por apresentar dificuldade de sucção e pouca abertura de boca, associados a malformações que deveriam ser investigadas pela genética.

Foi diagnosticado pela genética como portador da Síndrome de Pterígio Poplíteo por apresentar as seguintes características: sinéquias palpebrais e anquiloblefaro; bandas maxilo-mandibulares, fenda palatina posterior; sindactilia do $2^{\circ}$ e $3^{\circ}$ pododáctilos do pé direito; dificuldade de extensão completa do membro inferior direito (pterígio poplíteo) e estridor inspiratório leve.

O RN foi atendido na UTI neonatal no período de 26/11/2007 a 15/01/2008, fazendo uso de SNG até 11/01/2008 e iniciando, em 08/01/2008, a via oral (VO) complementada pela SNG. A mãe não tinha leite para amamentar.

O procedimento cirúrgico oftalmológico para ressecção das sinéquias palpebrais ocorreu em 30/11/2007. Um segundo procedimento cirúrgico foi realizado pela plástica, em 07/01/2008, para ressecção das bandas maxilo-mandibulares e frenotomia de lábio superior, com o objetivo de liberar a movimentação da mandíbula e aumentar a abertura de boca.

A avaliação fonoaudiológica ocorreu em 08/01/2008, no pós-operatório por solicitação da equipe médica. $\mathrm{O}$ RN encontrava-se na postura de decúbito dorsal, com estado de consciência alerta, tônus da musculatura global normal e boa estabilidade respiratória. Na avaliação das estruturas do SE, foram observados lábios com vedamento, língua no repouso no soalho da boca, língua na sucção e deglutição com canolamento e mobilidade funcional, bochechas simétricas e com coxins, mandíbula retraída com pouca abertura, gengivas superiores e inferiores hipertróficas, palato com fissura pósforame incisivo incompleta (classificação de Spina), reflexos orais de alimentação (busca, sucção, deglutição) presentes e adequados; reflexos de defesa (mordida, gag) anteriorizados e exacerbados. A paciente apresentou pouca tolerância ao toque intra-oral. A sucção não nutritiva (SNN), avaliada por meio da introdução do dedo mínimo enluvado na cavidade bucal do RN e por meio da chupeta ortodôntica, apresentou como resultado um número de sucções/pausas de 3:1, com ritmo presente, com força débil.

A primeira avaliação da sucção nutritiva $(\mathrm{SN})$, por meio da mamadeira, apresentou sucções/pausa de 5:1, pausas longas, demorando um tempo de $10 \mathrm{~min}$, aceitando $5 \mathrm{ml}$ dos $15 \mathrm{ml}$ prescritos. Foram observados sinais de estresse de alteração respiratória, estridor inspiratório leve, um episódio de engasgo e reflexo de gag anteriorizado e exacerbado, demonstrando hipersensibilidade oral em resposta ao bico convencional da mamadeira.

A intervenção fonoaudiológica foi realizada sempre antes da alimentação e consistia em estimular a região peri e intraoral feita com o dedo mínimo enluvado, ou com a chupeta, visando melhora das funções orais por meio da dessensibilização intra-oral, adequação dos reflexos de defesa, estimulação do reflexo de busca e abertura de boca, aumento e adequação do ritmo e pressão intra-oral e amadurecimento do reflexo de sucção e coordenação, com deglutição e respiração.

As mudanças observadas na paciente após as intervenções foram as seguintes: normalização da abertura de boca, adequação dos reflexos de mordida e gag, aumento do número de sucções para 12 por pausa, com força e coordenação adequadas, estabilidade respiratória e aceitação plena da dieta prescrita, em todos os horários.

Em 15/01/2008, o RN recebeu alta hospitalar, com via oral plena, na mamadeira com bico ortodôntico, aceitando toda a quantidade de alimentação prescrita e com peso de 3080 gramas.

\section{DISCUSSÃO}

Um dos principais objetivos da avaliação e intervenção fonoaudiológica com RN, na UTI Neonatal, é determinar de forma segura o momento adequado para a transição alimentar da sonda para a VO, permitindo que a alta não se demore por este fator $^{(1)}$.

O processo pode não acontecer de forma natural se o $\mathrm{RN}$ apresentar alterações anatômicas e funcionais, como as que foram identificadas no RN do presente caso, tais como: fissura palatal, hipersensibilidade oral e reflexo de mordida e gag exacerbados - alteração provocada, provavelmente, pelos procedimentos realizados no bebê, entre eles o uso da sonda, pois segundo estudos relatados na literatura, os lábios e a língua possuem uma grande quantidade de receptores sensoriais que, quando expostos a estímulos sensoriais negativos, resultantes de procedimentos como aspiração, intubação, cirurgias orais e uso de sondas oro ou nasogástricas, levam a hipersensibilidade oral. Esse fator pode determinar casos de recusa ou dificuldades alimentares, retardando o início da alimentação por $\mathrm{VO}$ e, portanto, a alta hospitalar ${ }^{(1,3,13)}$. É preciso ressaltar que o uso de sonda, no presente estudo, foi indicado pela recusa alimentar, que era provocada pela alteração dos reflexos orais e a impossibilidade de mobilidade labial e mandibular devido às bandas maxilo-mandibulares.

No presente caso, por meio da estimulação do sistema estomatognático e suas funções, com manuseio peri e intra-oral e uso da chupeta ortodôntica, foram realizadas as intervenções fonoaudiológicas com o objetivo de adequar a sensibilidade e 
reflexos orais alterados, organizar a coordenação das funções de deglutição com respiração e estimular a abertura de boca. Os manejos foram realizados com o $\mathrm{RN}$ em estado de alerta de forma organizada e por um período aproximado de cinco a dez minutos, antes da alimentação.

Estudos relataram os benefícios da estimulação oral como facilitador na transição da alimentação por sonda para a $\mathrm{VO}^{(12-}$ 13). A SNN, quando realizada antes da dieta leva a um melhor ganho de peso, uma vez que o RN estimulado mama todo o volume prescrito com maior facilidade, ativa o comportamento reflexo de procura, abertura de boca, sucção e deglutição, favorecendo a alimentação por mamadeira ou seio materno mais precocemente, facilitando o vínculo mãe-bebê e alta hospitalar precoce.

No estudo atual, a presença da fissura pós-forame e do estridor inspiratório associados à síndrome, acrescentam algumas particularidades à intervenção fonoaudiológica, uma vez que a pressão intra-oral estava prejudicada, aumentando a fadiga, o estridor e o tempo de alimentação. A criança portadora de fissura pós-forame ou transforame incisivo pode apresentar dificuldades alimentares por não conseguir uma pressão intraoral adequada. A sucção insuficiente, os vômitos e os engasgos ocorrem nos casos de falta de orientações adequadas à mãe, quanto ao melhor método de alimentar o recém-nascido. Estas orientações incluem o manejo da postura, de manobras de facilitação da sucção e deglutição, do tipo de bico e furo deste, como também o ritmo, com pausas coordenadas, para estabilizar a respiração, diminuindo a fadiga ${ }^{(1,3,13-14)}$.

Com respeito à postura, a cabeça deve estar em ângulo reto com o pescoço, bem apoiada, evitando-se que fique caída para trás ou fletida. Essa posição faz com que a gravidade traga a língua para frente. $\mathrm{O}$ bebê deve estar sentado, em posição de flexão, com os braços na linha média. No caso de presença de estridor inspiratório a literatura aponta como causa possível a laringomalácia que, em $85 \%$ dos casos, têm resolução espontânea até dois anos de idade. O manejo do estridor também prevê cuidados com a postura, já que pode aumentar na hora da alimentação, propiciando engasgos frequentes ${ }^{(15)}$.

O contato visual entre ele e a pessoa que o está nutrindo é muito importante, assim como a estimulação auditiva ${ }^{(1,3)}$. No presente caso, a mãe era muito receptiva às orientações, posicionando corretamente o bebê na alimentação e demonstrando um bom vínculo com a filha.

Após a alimentação, o bebê deve ser colocado no berço, com a cabeceira em posição mais elevada, em decúbito lateral direito. Essa posição auxilia na diminuição dos riscos de regurgitação, aspiração, otite média e aumenta o esvaziamento gástrico $^{(1)}$. Esta prática foi reforçada várias vezes para a mãe como parte da orientação também da alta.

Alguns autores afirmam que na fase inicial, e somente nesta fase, quando o recém-nascido fissurado ainda está apresentan- do alguma dificuldade para se adaptar à mamadeira, indicam-se manobras que facilitem o escoamento de leite para a cavidade oral, as quais estimulam o recém-nascido a desencadear a sucção tais como: apertar levemente o próprio bico da mamadeira nas laterais; pressionar o bico da mamadeira sobre a língua de forma a provocar o abaixamento da mandíbula; puxar levemente o bico da mamadeira para fora da boca; fazer pressão externa nas bochechas da criança, assim como embaixo de sua mandíbula. Esta última manobra melhora a pressão intra-oral por promover o vedamento labial mais eficiente ${ }^{(3,10-11,14)}$. Todas estas manobras foram implementadas na paciente com uma evolução favorável, mostrando-se eficazes.

Os bicos de mamadeira mais adequados são os ortodônticos por serem curtos e anatômicos. O furo deve ser graduado de acordo com a habilidade de sucção de cada bebê, sendo um tamanho regular usualmente indicado para intensificar o movimento de sucção. O bico é posicionado na região anterior da boca com o furo voltado para cima. Com o furo nessa posição não é direcionado para a cavidade nasal devido à postura vertical que favorece a ação da força gravitacional e escoamento do leite para a língua formar o bolo ${ }^{(3)}$. O uso do bico ortodôntico neste caso particular também facilitou a diminuição da exacerbação do reflexo de gag, apresentando o bebê uma melhor aceitação da alimentação com este utensílio.

Para bebês que apresentam alteração respiratória, fadiga e estridor, a literatura propõe a regulação do ritmo de sucção e do fluxo de leite. Fazer pausas mais frequentes para que recupere a ventilação, fracionar a dieta, posicionar adequadamente o paciente e manter um fluxo adequado são algumas das estratégias propostas que foram utilizadas ${ }^{(1)}$. Na medida em que o bebê em estudo foi realizando sua aprendizagem alimentar, a regulação do ritmo de sucções por pausas estabilizou-se em $12 \times 1$, permitindo a ingestão de toda a prescrição, num tempo de 15 minutos. A alteração respiratória diminuiu e a presença do estridor tornou-se cada vez mais discreta na alimentação, permanecendo mais evidente no choro.

\section{COMENTÁRIOS FINAIS}

Por meio da avaliação e intervenção fonoaudiológica neste caso foi possível detectar as alterações que poderiam levar a problemas de alimentação por via oral e permitiu uma evolução favorável, em pouco tempo, para a via oral plena e segura, possibilitando a alta hospitalar.

Cada dia mais, o manejo interdisciplinar de casos complexos como o discutido neste artigo, levam a refletir sobre o papel central da participação do fonoaudiólogo nas equipes de UTI Neonatal.

O fonoaudiólogo deve estar preparado para enfrentar desafios cada vez maiores, mesmo de patologias raras, como a do presente caso. 


\begin{abstract}
The aim of this study was to describe the speech-language intervention for the adequacy of the alimentary function through assessment and treatment of the stomatognathic system and its functions. The study was carried out based on the case report of a newborn with popliteal pterygium syndrome, fed by tube while taken care of at the Neonatal Intensive Care Unit (NICU). The speech-language assessment presented the following results: retracted jaw with little opening, hypertrofic inferior and superior gums, incomplete cleft palate, present and adequate oral reflexes (search, sucking, swallowing), anterior trigger of the gag reflex and overactive bite reflex. The oral sensitiveness was impaired. The non-nutritive sucking resulted in a ratio of 3:1 sucks per burst, with rhythm, but weak. In the nutritive sucking with a bottle, the newborn presented a 5:1 sucks per burst ratio, with long pauses. Signs of stress were observed, with breathing alteration, mild inspiratory stridor, a choking episode, and anterior and overactive gag reflex, demonstrating oral hypersensitivity in response to the use of a conventional bottle teat. Five weekly sessions of speech-language therapy were carried out, with orientations to the mother and the nursing team regarding position, oral stimulation and use of orthodontic bottle teats. The newborn was discharged with full oral feeding and good weight gain.
\end{abstract}

Keywords: Infant, newborn; Feeding behavior; Abnormalities, multiple; Cleft palate; Intensive care, neonatal

\section{REFERÊNCIAS}

1. Hernandez AM. O neonato de alto risco: proposta de intervenção global. In: Hernandez AM, organizador. Conhecimentos essenciais para atender bem o neonato. São José dos Campos: Pulso Editorial; 2003. p. 16-7.

2. Xavier C. Avaliação da alimentação de recém-nascido em fase de hospitalização (escala de avaliação motora oral da Irmandade Santa Casa de Misericórdia de São Paulo). Pró-Fono. 1995;7(2):69-74.

3. Pachi PR. Aspectos pediátricos. In: Altmann EBC. Fissuras labiopalatinas. 4a ed. Carapicuiba: Pró-Fono; 1997.

4. Bhering JA, Mendonça EG, Behering CA. Síndrome do pterígio poplíteo: uma visão pediátrica. An Hosp Sider Nac. 1985;9(4):181-4.

5. Fiorito LF, Carvalho AES. Síndrome de pterígio múltiplo: relato de caso. Pediatr Mod. 1985;20(7):373-4, 377-8.

6. Parashar SY, Anderson PJ, McLean N, Djohansjah M, David DJ. Spectrum of features in pterygium syndrome. Asian J Surg. 2006;29(2):104-8.

7. Deskin RW, Sawyer DG. Popliteal pterygium syndrome. Int J Pediatr Otorhinolaryngol. 1988;15(1):17-22

8. Reid J, Kilpatrick N, Reilly S. A prospective, longitudinal study of feeding skills in a cohort of babies with cleft conditions. Cleft Palate Craniofac J. 2006;43(6):702-9.
9. Reid J, Reilly S, Kilpatrick N. Sucking performance of babies with cleft conditions. Cleft Palate Craniofac J. 2007;44(3):312-20.

10. Bannister P. Conduta na alimentação inicial. In: Watson ACH, Sell DA, Grunwell P. Tratamento de fissura labial e fenda palatina. São Paulo: Santos; 2005.

11. Paradise JL, McWilliams BJ. Simplified feeder for infants with cleft palate. Pediatrics. 1974;53(4):566-8.

12. Amstalden-Mendes LG, Magna LA, Gil-da-Silva-Lopes VL. Neonatal care of infants with cleft lip and/or palate: feeding orientation and evolution of weight gain in a nonspecialized Brazilian hospital. Cleft Palate Craniofac J. 2007;44(3):329-34.

13. Fucile $\mathrm{S}$, Gisel E, Lau C. Oral stimulation accelerates the transition from tube to oral feeding in preterm infants. J Pediatr. 2002;141(2):230-6. Erratum in: J Pediatr 2002;141(5):743.

14. Araruna RC, Vendrúscolo DMS. Alimentação da criança com fissura de lábio e/ou palato: estudo bibliográfico. Rev Latinoam Enferm. 2000;8(2):99-105.

15. Avelino MAG, Liriano RYG, Fujita R, Pignatari S, Weckx LLM. O tratamento da laringomalácia: experiência em 22 casos. Rev Bras Otorrinolaringol. 2005;71(3):330-4.

Anexo 1. Ficha de levantamento de dados

Ficha de levantamento de dados

1. Identificação do RN

Nome da mãe:

Nome do RN:

DN:

sexo:

Data atual:

2. Manifestações do RN:

Apgar: 1' 5“

Comprimento:

Manobras de reanimação:

Uso de sonda:

Alimentação atual:
3. Resumo do caso:

\section{Diagnóstico do pediatra:}

Peso ao nascer:

Idade gestacional:

\section{Outras informações:}


Anexo 2. Avaliação fonoaudiológica

Avaliação fonoaudiológica

Identificação do paciente:

$\mathrm{RN}$ :

Data:

1. Observação pré-alimentação

Postura:

Estado de consciência:

Tônus da musculatura global:

Estabilidade respiratória:

\section{Estruturas do SSMO:}

\section{Lábios:}

fissura ( ) assimetria ( ) direita ( ) esquerda ( ) superior ( ) inferior ( ) tônus ( ) c/vedamento ( ) s/vedamento( )

\section{Língua no repouso:}

postura > retraída ( ) protuída ( ) soalho da boca ( ) ponta na papila ( ) elevação de dorso ( ) assimetrias ( )

Língua na sucção e deglutição:

canolamento ( ) mobilidade funcional ( )

mov. ântero-posterior ( ) protusão ( )

posteriorização ( ) clônus ( )

Bochecha:

simétricas ( ) assimétricas ( ) direita ( ) esquerda( )

colabadas ( ) coxins ( ) hipertônicas ( ) hipotônicas ( )

Mandíbula:

retraída ( ) simétrica ( ) assimétricas ( )

abertura exagerada ( ) tremores ( ) travamento ( )
Palato duro:

ogival ( ) atrésico ( ) fissura ( ) tipo:

\section{Reflexos orais:}

Busca:

Sucção:

Deglutição:

Mordida:

Gag:

\section{Sucção não-nutritiva:}

\section{Antes da alimentação:}

Sucções $x$ pausas:

Ritmo: presente ( ) desorganizado ( ) inconsistente ( )

Força: adequada( ) moderada( ) débil( ) exagerada( )

Pausas: rítmicas ( ) longas ( ) curtas ( )

Durante a alimentação com sonda:

Sucções $x$ pausas:

Ritmo; presente ( ) desorganizado ( ) inconsistente ( )

Força: adequada( ) moderada( ) débil( ) exagerada( )

Pausas: rítmicas ( ) longas ( ) curtas ( )

5. Sucção nutritiva:

Sucções x pausas:

Pausas: ausentes ( ) longas ( ) curtas ( ) adequadas ( )

Tempo de alimentação:

Sinais de estresse: escape de líquido ( ) alteração respiratória ( ) cianose ( ) variação de tônus global ( ) sonolência ( ) palidez ( )

Anexo 3. Ficha de observação da assistência à alimentação

Ficha de observação da assistência à alimentação

$\mathrm{RN}$ :

Localização na unidade:

Examinador:

\begin{tabular}{|l|l|l|l|}
\hline Data/hora & & & \\
\hline Idade & & & \\
\hline Peso & & & \\
\hline Estado geral & & & \\
\hline Assistência ventilatória e outros & & & \\
\hline Intercorrência do dia & & & \\
\hline $\begin{array}{l}\text { Alimentação SOG/SNG/VO } \\
\text { Outros }\end{array}$ & & & \\
\hline $\begin{array}{l}\text { Volume aceito } \\
\text { Volume prescrito }\end{array}$ & & & \\
\hline Ritmo de sucção não nutritiva 10“ & & & \\
\hline Ritmo de sucção nutritiva 10“ & & & \\
\hline Mililitros sugados 15، & & & \\
\hline Estimulação intra-oral & & & \\
\hline Outros procedimentos & & & \\
\hline Observações & & & \\
\hline
\end{tabular}

